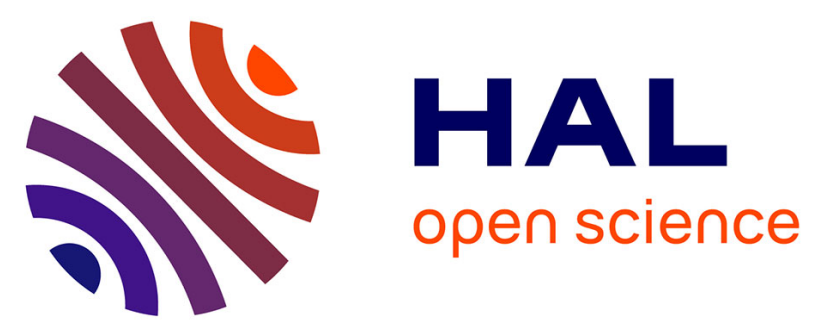

\title{
Do We Need Inhibitory Control to Be Creative? Evidence From a Dual-Task Paradigm.
}

Anaëlle Camarda, Gregoire Borst, Marine Agogué, Marianne Habib, Benoit Weil, Olivier Houde, Mathieu Cassotti

\section{- To cite this version:}

Anaëlle Camarda, Gregoire Borst, Marine Agogué, Marianne Habib, Benoit Weil, et al.. Do We Need Inhibitory Control to Be Creative? Evidence From a Dual-Task Paradigm.. Psychology of Aesthetics, Creativity, and the Arts, In press, 12 (3), pp.351-358. 10.1037/aca0000140 . hal-01761381

\section{HAL Id: hal-01761381 \\ https://hal.science/hal-01761381}

Submitted on 9 Apr 2018

HAL is a multi-disciplinary open access archive for the deposit and dissemination of scientific research documents, whether they are published or not. The documents may come from teaching and research institutions in France or abroad, or from public or private research centers.
L'archive ouverte pluridisciplinaire HAL, est destinée au dépôt et à la diffusion de documents scientifiques de niveau recherche, publiés ou non, émanant des établissements d'enseignement et de recherche français ou étrangers, des laboratoires publics ou privés. 
Do we need inhibitory control to be creative? Evidence from a dual-task paradigm.

\section{Anaëlle Camarda}

CNRS Unit 8240, Laboratory for the Psychology of Child Development and Education, Paris

Descartes University, Sorbonne Paris Cité University and Caen University, France

Grégoire Borst

Institut Universitaire de France

\& CNRS Unit 8240, Laboratory for the Psychology of Child Development and Education, Paris

Descartes University, Sorbonne Paris Cité University and Caen University, France

Agogué Marine

Descartes University, Sorbonne Paris Cité University and Caen University, France

Habib Marianne

Paragraph Laboratory (EA349), Paris 8 university, France

Benoit Weil

Center for Management Science, Chair TMCI, Mines ParisTech, France

Olivier Houdé

Institut Universitaire de France

\& CNRS Unit 8240, Laboratory for the Psychology of Child Development and Education, Paris

Descartes University, Sorbonne Paris Cité University and Caen University, France

Mathieu Cassotti

Institut Universitaire de France

\& CNRS Unit 8240, Laboratory for the Psychology of Child Development and Education, Paris Descartes University, Sorbonne Paris Cité University and Caen University, France

Acknowledgment : This research was financed by a grant from the French National Research Agency (ANR-13-SOIN-0004-02 IDéfixE). 


\begin{abstract}
The ability to inhibit common and dominant paths of solutions to a problem seems to be a critical process for generating creative ideas. However, previous behavioral studies have not systematically supported a positive relation between creativity and inhibitory control. Thus, the purpose of the present study was to determine the potential role of inhibitory control in creative idea generation. In experiment 1 , we used a dual-task paradigm to reduce participants' inhibitory control resources while performing a creative task. Participants were asked to propose as many creative solutions as possible to prevent a hen's egg from breaking when dropped from a height of $10 \mathrm{~m}$ under either interference or control conditions of a computerized version of the Color-Word Stroop task. We found that inhibitory control load decreased creative capabilities in terms of fluidity and expansivity. To determine whether creative idea generation depends specifically on the ability to inhibit fixation effects, dual-task costs under a secondary working memory (WM) task were examined in a second experiment. The results revealed that WM load had no significant effect on creative ideation. Combined, these results confirmed that inhibitory control is a core process to overcoming fixation effects and generating original solutions in a creative task.
\end{abstract}

(C) 2017, American Psychological Association. This paper is not the copy of record and may not exactly replicate the final, authoritative version of the article. Please do not copy or cite without authors permission. The final article will be available, upon publication, via its DOI: 10.1037/aca0000140 
Do we need inhibitory control to be creative? Evidence from a dual-task paradigm

The ability to inhibit common and dominant paths of solutions to a problem seems to be critical to generating creative ideas (Cassotti, Agogué, Camarda, Houdé \& Borst, 2016; Dietrich \& Kanso, 2010). Although psychological and neuroimaging studies have both shown that inhibitory control is a core process involving numerous cognitive domains including reasoning (Houdé \& Borst, 2014; 2015), decision making (Crone \& Dahl, 2012) and theory of mind (Bull, Phillips \& Conway, 2007), experimental studies to date have provided discrepant results regarding its role in creativity (Radel, Davranche, Fournier, \& Dietrich, 2015; Beaty, Silvia, Nusbaum, Jauk, \& Benedek, 2014). While some studies report that generating creative solutions to a problem requires the inhibition of previous inappropriate ideas inducing fixation phenomena (see Cassotti et al., 2016 for a review), other studies suggest that inhibitory control hinders creative potential (Radel et al., 2015). Therefore, the present study aimed to clarify the potential role of inhibitory control in creative idea generation.

Early models of creativity assumed that creativity involved automatic processes such as loose associations and disinhibition (Martindale, 1999; Eysenck, 1995). According to this view, a lack of inhibitory control would be beneficial to fostering remote associations and intuitive thinking, leading to a stimulation of creative ideation. Empirical support of the assumption that poorer inhibitory ability facilitates creativity was provided by studies that showed that performances on inhibitory control tasks were negatively correlated to creative idea generation based on divergent thinking measures (Dorfman, 2008; Kharkhurin, 2011; Lien \& Lien, 2013a). The deleterious effect of inhibition on creativity was also supported by clinical studies of patients 
exhibiting inhibitory control deficits. For example, patients with ADHD and bipolar disorder provided more original associations and creative ideas in several creative tasks than healthy participants (Abraham et al., 2006; Healey \& Rucklidge, 2006; Reverberi, Toraldo, D’Agostini \& Skrap, 2005; Russ, 2001; White \& Shah, 2006). However, it should be noted that these patients rarely exhibited specific deficits in inhibitory control and that other investigations of patients with clinical disorders have provided discrepant results (see de Souza, Guimarães, Teixeira, Caramelli, Levy, Dubois, \& Volle, 2014).

Additional evidence of the negative role of inhibitory control in creative thinking was provided by a study that asked participants to solve a creative task in which they had to generate unusual and creative uses of conventional objects, such as a brick, after performing inhibitory control tasks designed to exhaust their inhibitory control resources (Radel, Davranche, Fournier \& Dietrich, 2015). In this study, the depletion of inhibitory control resources enhanced both the fluency and the originality of the ideas proposed by the participants, suggesting that "disinhibition" stimulates creativity.

In sharp contrast with the aforementioned studies, an increasing number of studies demonstrate a positive role of inhibitory control during idea generation. For example, a series of correlational studies have reported that inhibitory control performance, assessed using seminal inhibitory control tasks such as the Color Word Stroop task (Stroop, 1935), are positively related to various creative measures in adults (Beaty, Silvia, Nusbaum, Jauk, \& Benedek, 2014; Benedek, Franz, Heene, \& Neubauer, 2012; Vartanian, 2009). Additionally, industrial designers, who are experts in the creative exploration of alternative ways of thinking, exhibit not only higher divergent thinking ability but also higher inhibitory control efficiency compared to a control group of participants (Edl, Benedek, Papousek, Weiss, \& Fink, 2014). 
Some of the strongest evidence linking inhibitory control to creative thinking has been provided by neuroimaging studies that have shown a positive relationship between the ability to generate highly creative solutions to a problem and activation of specific prefrontal brain regions known to be implicated in executive function and inhibitory control in particular (Benedek et al., 2014; Dietrich \& Kanso, 2010). For instance, activation within the inferior frontal gyrus - a brain region classically associated with inhibitory control (Aron, Robbins, \& Poldrack, 2004; 2014; Houdé, Rossi, Lubin, \& Joliot, 2010)—was positively related to the originality and appropriateness of ideas proposed by participants (Benedek et al., 2014). These results are in line with a meta-analysis of 45 FMRI studies on creative thinking, which showed that verbal and visuospatial creativity activated regions in the prefrontal network including the anterior cingulate cortex, the inferior frontal gyri, and the middle frontal gyri (Boccia, Piccardi, Palermo, Nori, \& Palmiero, 2015), three structures involved in conflict monitoring, inhibitory control, and working memory, respectively. Thus, these three processes might have a fundamental role in creative ideation.

The hypothesis that inhibitory control is a core component of creative thinking is further supported by studies showing that previously acquired and existing knowledge or ideas can limit creative idea generation, leading to mental fixation (Storm \& Angello, 2010; Storm \& Patel, 2014). While creative tasks require the exploration of new and original solutions, individuals tend to follow "the path of least resistance" and propose solutions based on common and undemanding design heuristics (Agogué, Poirel, Pineau, Houdé, \& Cassotti, 2014; Finke, Ward, \& Smith, 1992). For example, when individuals must design methods to ensure that a hen's egg will not break when dropped from a height of 10 meters ( $32 \mathrm{feet})$, the results revealed that adults fixed on a limited number of response categories based on the most accessible knowledge 
(Cassotti, Camarda, Poirel, Houdé, \& Agogué, 2016). Most of the solutions provided by the participants consisted of using an inert device to dampen the shock, protect the egg or slow the fall (e.g., to slow the fall with a parachute), whereas more original categories of solutions that consisted of using a living object or modifying the natural properties of the egg (e.g., training a bird to catch the egg during the fall or freezing the egg before dropping it) were proposed less often by the participants. This "dark side" of fast and intuitive strategies to creatively solving a problem has led to the recent development of a dual process model of creative idea generation (Cassotti et al., 2016a). Following this view, creative idea generation requires the inhibition of dominant and common ideas within an intuitive and heuristic System 1 to explore new concepts with a generative type of reasoning within a deliberate and analytic System 2. Thus, to provide original ideas to problems such as "the egg task", one must first inhibit the intuitive and dominant paths to solutions that create fixation effects (referring to the first System) and then activate conceptual expansion reasoning (referring to the second System)

The discrepancies observed regarding the potential role of inhibitory control in creativity underscore the need to design experiments that systematically manipulate inhibitory control resources. Thus, in the present study, we aim to test whether inhibitory control is a critical process to generating multiple creative ideas by using a dual-task paradigm in which participants are asked to perform a creative task while performing an inhibitory control task. Dual-task methodology has proven useful for testing the involvement of executive resources in various domains such as reasoning (De Neys, 2006a; 2006b) and theory of mind (Bull, Phillips \& Conway, 2007). In the present study, the participants performed a verbal creativity task (i.e., the egg task, Agogué et al., 2014, Cassotti et al., 2016) in which they had to propose strategies to dropping a hen's egg from a height of $10 \mathrm{~m}$ without breaking it while performing no task (single 
task) or the congruent (control dual task) or incongruent (inhibition dual task) conditions of the Color Word Stroop task. Indeed, the Color Word Stroop task has been proven to be effective in reducing inhibitory control resources in dual-task paradigms in previous studies (e.g., Brown, Collier, \& Night, 2013). In both conditions, the participants had to identify the ink colors of printed words that denoted different colors. In the congruent condition, the ink color was congruent with the color denoted by the words (e.g., BLUE printed in blue), whereas in the incongruent condition, the ink color was incongruent with the color denoted by the words (e.g., BLUE printed in red). Although both conditions require attentional resources, only the incongruent one requires inhibitory control to avoid reading the word meaning instead of the ink color.

Importantly, this dual-task paradigm allows us to test contrasting predictions based on the contradictory theoretical views regarding the role of inhibitory control in creative thinking.

We reasoned that if creative idea generation operates automatically and inhibitory control hinders creativity as suggested by Radel et al. (2015), then participants should be more creative (as indicated by better performance on the verbal creativity task) during the incongruent conditions of the Color Word Stroop task (i.e. inhibition dual task inducing a "disinhibition") than participants performing the verbal creativity task under the single-task condition (i.e. in which inhibitory control resources are fully available). On the other hand, if creative idea generation draws on inhibitory control resources as suggested by the dual process approach (Cassotti et al., 2016), participants should be less creative (as indicated by lower performance on the verbal creativity task) during the incongruent conditions of the Color Word Stroop task than participants in the single-task condition. Finally, participants should be as creative during the congruent condition of the Color Word Stroop task (control dual task) as participants performing 
the verbal creativity task in the single-task condition.

\section{Experiment 1}

Method

\section{Participants}

Seventy-eight undergraduate students (55 females, 23 males, mean age $=20.49$ years old, range $=18$ years to 32 years, $S D=2.15$ ) from Paris Descartes University participated in this study. All participants reported normal or corrected-to-normal vision. Each participant was randomly assigned to one of three experimental conditions: an inhibition dual-task condition $(n=$ 28), a control dual-task condition $(n=24)$ and a single-task condition in which participants performed the creative task without a secondary task load $(n=26)$. The mean age did not differ between the three groups of participants assigned to the three conditions, $F(2,75)=1.97, p=.15$. All of the participants provided written consent and were tested in accordance with national and international norms governing the study of human research participants.

\section{Design and Procedure}

Regardless of the experimental conditions, participants performed a creative task in which they were given five minutes to propose as many original solutions as possible to the following problem: "Ensure that a hen's egg does not break when dropped from a height of 10 m." (Agogué et al., 2014a; 2014b; 2015; Cassotti et al., 2016). Participants were instructed that there were no right or wrong answers and that they had to provide as many creative solutions to the problem as possible. Critically, they were asked to provide their answer as soon as they came to their mind. 
To facilitate idea generation, the participants were seated alone in the experimental room, and they had to provide oral responses (recorded by a Dictaphone) to the egg task

Participants' responses were recorded throughout the task. To measure the effect of the dual-task condition on creativity in the egg task, we evaluated the participants' answers based on three criteria: fluidity (the ability to generate many solutions, as measured by the number of solutions), flexibility (the ability to generate many categories of solutions), and expansivity (the ability to provide solutions outside the fixation effect).

More specifically, to measure fluidity, we counted the number of solutions provided by the participants. When a participant proposed a solution that combined different proposals, we counted each proposal as one solution. With regard to flexibility, a trained rater assigned each solution to one of 54 solution categories (e.g., "using a pool of water on the floor to reduce the shock"). Subsequently, the number of applied solution categories was counted for each participant. We applied a well-validated measurement of originality on the egg task (i.e., expansivity) by studying the distribution of solutions in different categories. To do so, a trained rater assigned each solution given by the participant to one of 10 meta-categories (Agogué et al., 2014a; 2014b; 2015; Cassotti et al., 2016). Based on previous studies, three meta-categories (i.e., reducing the shock, protecting the egg, and slowing the fall) met the qualifications for the fixation effect, whereas the other seven did not (e.g., using a living object and modifying the natural properties of the egg). To assess expansivity, we then counted the number of solutions provided that were outside the fixation effect for each participant. Critically, this qualitative measure of creativity is highly correlated with expert evaluations of the ideas using consensual assessment (Agogué et al., 2015; Amabile, Goldfarb, \& Brackfleld, 1990). 
In both dual-task conditions, the participants completed the egg task concurrently with either the congruent (i.e., the control dual-task condition) or the incongruent (i.e., the inhibition dual-task condition) version of the Color Word Stroop task. In both conditions, the participants were tested using laptop computers with a screen resolution of $1366 * 768$ pixels, $(310 * 170 \mathrm{~mm} 2)$. Stimuli were presented using E-Prime 2.0 software (Psychology Software Tools, Pittsburgh, PA).

\section{(FIGURE 1)}

In the congruent and incongruent conditions of the Color Word Stroop task, participants were asked to identify the ink colors of printed words that denoted colors. In the incongruent condition, the ink color and the color meaning of the word were incongruent (e.g., red printed in green ink, see Figure 1), and thus the participants had to inhibit the meaning of the word (e.g., red) to correctly identify the ink color (e.g., green). In the congruent condition, inhibition was not required because the ink colors and the colors denoted by the words were congruent (e.g., red printed in red ink). Nine Stroop items were created by combining different color names ('red', 'green', 'blue') with the three corresponding ink colors (RGB color codes 255;0;0, 0;255;0, and 0;0;255). Three items were congruent (e.g., 'red' written in red) and 6 were incongruent (e.g., 'red' written in blue). The words were presented on the screen in 24- pt Courier New bold type on a black background. In both conditions, participants provided their responses by pressing one of three keyboard buttons associated with the three possible ink colors (i.e., "red", "green", and "blue"). Participants were asked to answer as quickly as possible while maintaining high accuracy. Therefore, participants provided oral responses to the Egg task and concurrently provided motor responses to the Stroop items by pressing one of three keyboard buttons. Each condition involved 141 experimental trials preceded by a training session of 57 randomly ordered 
trials. To control that each participant performed the same number of trials in each condition, each item (i.e., incongruent stimuli for the inhibition dual-task condition and congruent stimuli for the control dual-task condition) remained on the screen during $1300 \mathrm{~ms}$ and was preceded by a white fixation cross displayed on a black background for $330 \mathrm{~ms}$. After each trial, they received feedback on the accuracy of their responses for $500 \mathrm{~ms}$. In each trial of the training session, a colored dot was displayed in the center of the screen, and the participants pressed the corresponding response button as quickly as possible. The training session aimed to automatize the participants' motor response. Finally, to ensure that the participants actually performed the Color Word Stroop task while generating creative ideas, we excluded participants with a performance in the congruent or incongruent conditions of the Color Word Stroop task that was lower than two median absolute deviations (MADs) from the median of the group. Thus, two participants from the inhibition dual-task condition and one participant from the control dual-task condition were excluded from the subsequent analysis.

Results

The fluidity, flexibility and expansivity scores were submitted to one-way analyses of variance (ANOVAs) with condition as the between-subject factor (inhibition dual-task condition, control dual-task condition and single-task condition), and we used partial eta squared $\left(\eta_{\mathrm{p}}{ }^{2}\right)$ and Cohen's $d$ to assess the effect size. Correlation analysis between our different measures of creativity revealed that fluidity and flexibility scores were highly correlated, $r(73)=.82, p<.01$. In addition, a significant correlation was found between fluidity and expansivity scores, $r(73)=$ $.61, p<.01$. Given that the correlation between the fluidity and flexibility scores was higher than 
.80 , we have restricted the data analysis to the fluidity and expansivity scores to avoid redundancy.

(FIGURE 2)

Regarding fluidity, the one-way ANOVA revealed a main effect of condition, $F(2,72)=$ 3.52, $\mathrm{p}=.03, \eta_{p}{ }^{2}=.09$ (see Figure $\left.2 \mathrm{~A}\right)$. More specifically, the planned contrasts revealed that the participants in the inhibition dual-task condition $(M=7.04, S D=5.17)$ proposed fewer solutions than those in the single-task $(M=10.69, S D=4.91), F(1,72)=5.75, p=.02, d=.72$, and the control dual-task condition and the control dual-task conditions $(M=10.43, S D=6.4), F(1,72)$ $=4.66, p=.03, d=.58$. In addition, the results showed no significant difference between the participants in the control dual-task condition $(M=10.43, S D=6.40)$ and those in the single-task condition in terms of fluidity $(M=10.69, S D=4.91), F(1,72)<1, d=.05$. Regarding expansivity, the main effect of condition tended to reach significance, $F(2,72)=2.56, p=.08$, $\eta_{p}{ }^{2}=.07$. Given our theory-driven hypothesis, we examined the differences between the three conditions using independent t-tests. The results showed that participants in the inhibition dualtask condition provided fewer responses outside the fixation paths (i.e., expansivity, $M=1.73$, $S D=1.87)$ than those in the single-task condition $(M=3.12, S D=2.64), t(50)=2.18, p=.03, d$ $=.61$. In addition, the results showed no significant differences between the participants in the single-task condition and those in the control dual-task condition $(M=2.82, \mathrm{SD}=2.38), t(57)=$ $0.40, p=.69$.

Discussion

Using a dual-task paradigm that manipulated the availability of inhibitory control 
resources during a creative idea generation task, we observed that inhibitory control load decreased creative capabilities in terms of fluidity and expansivity. In sharp contrast with the idea that disinhibition stimulates creativity (Radel et al., 2015) and in line with previous neuroimaging and behavioral studies (Beaty, Silvia, Nusbaum, Jauk, \& Benedek, 2014; Benedek, Franz, Heene, \& Neubauer, 2012; Vartanian, 2009), our findings supported a dual process model of creativity according to which inhibitory control is required to overcome fixation effects in idea generation (Cassotti et al., 2016). Although our results clearly demonstrated the involvement of cognitive control in creativity, one limitation of the present study might be that our findings failed to determine whether the dual-task cost depended specifically on inhibitory control or whether it resulted from a more general executive control cost.

To determine whether creative idea generation depends specifically on the ability to inhibit fixation effects, the dual-task costs under a secondary working memory (WM) task were examined in a second experiment. We reasoned that if the generation of creative solutions in the egg task depended specifically on the ability to inhibit fixation effects, creativity should not decrease under the secondary WM task load. In contrast, if creative idea generation requires broader executive function resources, then creativity should also be impaired under the secondary WM task load. Finally, if working memory is detrimental to creativity as suggested by Lin and Lien (2013b), creative performance should be higher under the secondary high-demanding WM task load. Indeed, in line with the hypothesis that creative idea generation operates automatically, Lin and Lien (2013b) have previously showed that depleting participants' working memory resources using dual task paradigm increases the fluidity in a divergent thinking task.

\section{Experiment 2}


Method

\section{Participants}

A new sample of seventy-nine undergraduate students (57 females, 22 males, mean age = 21.03 years old, range $=18$ years to 32 years, $S D=2.64)$ from Paris Descartes University participated in this experiment. Each participant was randomly assigned to one of three experimental conditions: a high-demanding WM dual-task condition $(n=25)$, a low-demanding WM control dual-task condition $(n=25)$ and a single-task condition in which participants performed the creative task without a secondary task load $(n=29)$. The mean age did not significantly differ between the three conditions, $F(1,76)<1$. All participants reported normal or corrected-to-normal vision. All of the participants provided written consent and were tested in accordance with national and international norms governing the study of human research participants.

\section{Design and Procedure}

Regardless of the experimental conditions, participants performed the egg task (see Experiment 1) in which they were given five minutes to propose as many original solutions as possible (Agogué et al., 2014a; 2014b; 2015; Cassotti et al., 2016). In both dual-task conditions, they were asked to provide their answer orally as soon as they came to their mind while performing either a computerized high-demanding WM task (i.e., the WM dual-task condition) or a computerized low-demanding WM task (i.e., the control dual-task condition). In both conditions, the participants were tested using laptop computers with a screen resolution of 
$1366 * 768$ pixels $(310 * 170 \mathrm{~mm} 2)$. Stimuli were presented using E-Prime 2.0 software (Psychology Software Tools, Pittsburgh, PA).

(FIGURE 3)

In both dual-task conditions, the participants were required to complete the "dot memory task" (De Neys, 2006a; 2006b). In the dot memory task, participants were instructed to memorize the sequence of presentation of four dots in a $4 \times 4$ matrix (See Figure 3). Each dot was presented during $1000 \mathrm{~ms}$. After the presentation of the fourth dot, an orange matrix was displayed for 1000 ms before the presentation of an empty matrix. As soon as the empty matrix was displayed, participants were asked to reproduce the pattern of dot locations by selecting with the mouse the successive location of the dots on the empty matrix displayed on the screen. Critically, in the WM load condition, the matrix contained complex four-dot patterns, whereas in the control load condition, the patterns consisted of four dots on a horizontal or diagonal line. Each condition involved 22 experimental trials that were randomly presented. To control that each participant performed the same number of trials in each condition, the empty response matrix remained on the screen until the participant responded, within a time limit of $7000 \mathrm{~ms}$. After each trial, they received feedback on the accuracy of their responses. The duration of the feedback ranged from $1000 \mathrm{~ms}$ to $7000 \mathrm{~ms}$ depending of the response time of the participant in such a way that cumulate duration of the response matrix and the feedback reaches $8000 \mathrm{~ms}$ in total on each trial. To ensure that the participants performed the dual task, we excluded those with a performance on the WM tasks that was lower than two MADs from the median of the group. Thus, one participant from the control dual-task condition was excluded from the subsequent analysis.

To determine whether the high-demanding WM task requires additional WM resources than the low-demanding WM task, we conducted a control study on 21 participants (4 men, 17 
women, mean age $=20.80$ years old, $S D=1.88$ ). Participants completed both tasks and the order of presentation of the tasks was counterbalanced across participants. As expected, participants required less time to perform the low-demanding WM trials $(M=1649 \mathrm{~ms}, S D=337.2 \mathrm{~ms})$ than the high-demanding WM trials $(M=1867 \mathrm{~ms}, S D=406.8 \mathrm{~ms}), t(20)=2,82, p=0.01, d=0.61$. In addition, participants were less accurate (i.e. Accuracy Rate) in the high-demanding WM task ( $M$ $=82 \%, S D=13 \%)$ than in the low-demanding WM task $(M=93 \%, S D=7 \%), t(20)=4,48, p<$ $0.001, d=0.97$. Thus, we are confident that the high-demanding WM task requires additional WM resources than the low-demanding WM Task

Results

The fluidity, flexibility and expansivity scores were submitted to one-way analyses of variance (ANOVAs) with condition as $a$ between-subject factor (a high-demanding WM dualtask condition, a low-demanding WM control dual-task condition and a single-task condition). The analysis revealed no significant main effects of condition for fluidity, $F(2,75)<1$ (see Figure 2B), or expansivity, $F(2,75)<1$. Regarding fluidity, further independent t-tests confirmed the lack of significant difference between participants in the high-demanding WM dual-task condition $(M=11.08, S D=5.73)$ and those in the single-task condition $(M=10.45, S D=5.36)$, $t(52)=0.42, p=.68$. Similar results were obtained for the expansivity measures. The analysis revealed no significant differences between participants in the WM dual-task condition (expansivity: $M=5.24, S D=4.31)$ and those in the single-task condition $(M=5, S D=2.45, t(52)$ $=0.25, p=.81)$.

General discussion

The purpose of the present study was to determine the potential role of inhibitory control 
in creative idea generation. Two major findings emerged from this investigation: 1) Participants' ability to provide creative ideas decreased under inhibitory control load (Experiment 1), whereas 2) WM load had no significant effect on creative ideation (Experiment 2). Taken together, these results confirm that inhibitory control is critical to overcoming fixation effects and generating original solutions in a creative task. Moreover, our results extend the findings of previous correlational studies by revealing a more causal link between the availability of inhibitory control resources and creative capabilities (Beaty, Silvia, Nusbaum, Jauk, \& Benedek, 2014; Benedek, Franz, Heene, \& Neubauer, 2012; Vartanian, 2009). In contrast with the assumption that “disinhibition" and reduced WM resources foster remote associations and stimulate creativity (Radel, Davranche, Fournier, \& Dietrich, 2015; Lin and lien, 2013a; 2013b), the results of both experiment 1 and experiment 2 did not find that inhibitory control or WM load had any stimulation effects. However, in agreement with the dual process model of creativity (Cassotti et al., 2016), the ability to inhibit intuitive-heuristic thinking (System 1) leading to fixation seemed fundamental to generating creative ideas by allowing individuals to adopt other types of system 2 reasoning (e.g., analogical thinking and conceptual expansion).

An alternative interpretation of the absence of an effect of working memory load on creativity might be that the working memory task was too easy when compared to the interference condition of the Color Word Stroop task. However, this hypothesis seems less likely, as the task used in the present study has been proven to be effective in reducing WM resources in dual-task paradigms for other domains such as reasoning and decision making (see, for example, Bago, \& De Neys, 2017; De Neys, 2006a; 2006b). In addition, even if this task only require to store information and does not require the manipulation of the information per se, we note that the dot matrix task seems to tax executive processes as suggested by the correlations observed 
between this task and the tower of Hanoi or the random number generation task, two classical executive function tasks (Miyake, Friedman, Rettinger, Shah, \& Hegarty, 2001). However, further studies are needed to determine whether a more executive demanding version of the dot memory task requiring for example to recall dots in backward order might negatively influence creative ideas generation.

\section{Conclusion}

The present study is the first to demonstrate a cost of inhibitory control load during creative idea generation using a dual-task paradigm. Our results clearly suggest that not all executive functions are involved in creative thinking and that inhibitory control is a core process of creative ideation. Accordingly, the present study provides new evidence for the current debate on the role of inhibitory control in creative idea generation. 


\section{References}

Abraham. A., Windmann. S., Siefen. R., Daum. I., \& Güntürkün, O. (2006). Creative thinking in adolescents with attention deficit hyperactivity disorder (ADHD). Child Neuropsychology, 12, $111-123$.

Agogué, M., Le Masson, P., Dalmasso, C., Houdé, O., \& Cassotti, M. (2015). Resisting Classical Solutions: The Creative Mind of Industrial Designers and Engineers. Psychology of Aesthetics, Creativity, and the Arts, 9, 313-318.

Agogué, M., Kazakçi, A., Hatchuel, A., Masson, P., Weil, B., Poirel, N., \& Cassotti, M. (2014a). The impact of type of examples on originality: Explaining fixation and stimulation effects. The Journal of Creative Behavior, 48, 1-12.

Agogué, M., Poirel, N., Pineau, A., Houdé, O., \& Cassotti, M. (2014b). The impact of age and training on creativity : a design-theory approach to study fixation effects. Thinking Skills and Creativity, 11, 33-41.

Amabile, T. M., Goldfarb, P., \& Brackfleld, S. C. (1990). Social influences on creativity: Evaluation, coaction, and surveillance. Creativity research journal, 3, 6-21.

Aron, A. R., Robbins, T. W., \& Poldrack, R. A. (2004). Inhibition and the right inferior frontal cortex. Trends in cognitive sciences, 8, 170-177.

Aron, A. R., Robbins, T. W., \& Poldrack, R. A. (2014). Inhibition and the right inferior frontal cortex: one decade on. Trends in cognitive sciences, 18, 177-185.

Bago, B., \& De Neys, W. (2017). Fast logic?: Examining the time course assumption of dual process theory. Cognition, 158, 90-109. 
Beaty, R. E., Silvia, P. J., Nusbaum, E. C., Jauk, E., \& Benedek, M. (2014). The role of associative and executive processes in creative cognition. Memory \& Cognition, 42, 1186-1197.

Benedek, M., Franz, F., Heene, M., \& Neubauer, A. C. (2012). Differential effects of cognitive inhibition and intelligence on creativity. Personality and Individual Differences, 53, $480-485$.

Benedek, M., Jauk, E., Fink, A., Koschutnig, K., Reishofer, G., Ebner, F., et al. (2014). To create or to recall? Neural mechanisms underlying the generation of creative new ideas. NeuroImage, 88, 125-133.

Boccia, M., Piccardi, L., Palermo, L., Nori, R., \& Palmiero, M. (2015) Where do bright ideas occur in our brain? Meta-analytic evidence from neuroimaging studies of domain- specific creativity. Frontiers in Psychology, 6:1195.

Brown, S. W., Collier, S. A., \& Night, J. C. (2013). Timing and executive resources: dualtask interference patterns between temporal production and shifting, updating, and inhibition tasks. Journal of experimental psychology: human perception and performance, 39, 947.

Bull, R., Phillips, L. H., \& Conway, C. A. (2008). The role of control functions in mentalizing: Dual-task studies of theory of mind and executive function. Cognition, 107, 663672.

Cassotti, M., Agogué, M., Camarda, A., Houdé, O., \& Borst, G. (2016a). Inhibitory Control as a Core Process of Creative Problem Solving and Idea Generation from Childhood to Adulthood. New directions for child and adolescent development, 151, 61-72. 
Cassotti, M., Camarda, A., Poirel, N., Houdé, O., \& Agogué, M. (2016b). Fixation effect in creative ideas generation: Opposite impacts of example in children and adults. Thinking Skills and Creativity, 19, 146-152.

De Neys, W. (2006a). Dual processing in reasoning: Two systems but one reasoner. Psychological Science, 17, 428-433.

De Neys, W. (2006b). Automatic-heuristic and executive-analytic processing in reasoning: Chronometric and dual task considerations. Quarterly Journal of Experimental Psychology, 59, 1070-1100.

de Souza, L. C., Guimarães, H. C., Teixeira, A. L., Caramelli, P., Levy, R., Dubois, B., \& Volle, E. (2014). Frontal lobe neurology and the creative mind. Frontiers in psychology, 5.

Dietrich, A., \& Kanso, R. (2010). A review of EEG, ERP, and neuroimaging studies of creativity and insight. Psychological bulletin, 136, 822-848.

Dorfman, L., Martindale, C., Gassimova, V., \& Vartanian, O. (2008). Creativity and speed of information processing: a double dissociation involving elementary versus inhibitory cognitive task. Personality and individual differences, 44, 1382 - 1390.

Eysenck, H. J. (1995). Genius. The natural history of creativity. Cambridge, UK: Cambridge University Press.

Finke, R.A., Ward, T.B., \& Smith, S.M. (1992). Creative cognition: Theory, research, and applications. Cambridge, MA: MIT Press.

Healey. D., \& Rucklidge. J. J. (2006). An investigation into the relationship among 
ADHD symptomatology, creativity, and neuropsychological functioning in children. Child Neuropsychology, 12, $421-438$.

Houdé, O., \& Borst, G. (2014). Measuring inhibitory control in children and adults. Frontiers in Psychology, 5:616.

Houdé, O., \& Borst, G. (2015) Evidence for an inhibitory-control theory of the reasoning brain. Frontiers in Human Neuroscience, 9:148.

Houdé, O., Rossi, S., Lubin, A., \& Joliot, M. (2010). Mapping numerical processing, reading, and executive functions in the developing brain: an fMRI meta- analysis of 52 studies including 842 children. Developmental science, 13, 876-885.

Lin, W. L., \& Lien, Y. W. (2013a). Exploration of the relationships between retrievalinduced forgetting effects with open-ended versus closed-ended creative problem solving. Thinking skills and creativity, 10, $40-49$.

Lin, W. L., \& Lien, Y. W. (2013b). The different role of working memory in open-ended versus closed-ended creative problem solving : a dual-process theory account. Creativity research journal, 25, $85-96$.

Martindale, C. (1999). Biological bases of creativity. In R. J. Sternberg (Ed.), Handbook of creativity (pp. 137-152). Cambridge, U.K.: Cambridge University Press.

Miyake, A., Friedman, N. P., Rettinger, D. A., Shah, P., \& Hegarty, M. (2001). How are visuospatial working memory, executive functioning, and spatial abilities related? A latentvariable analysis. Journal of experimental psychology: General, 130, 621. 
Radel, R., Davranche, K., Fournier, M., \& Dietrich, A. (2015). The role of (dis) inhibition in creativity: Decreased inhibition improves idea generation. Cognition, 134, 110-120.

Reverberi, C., Toraldo, T., D’Agostini, S., \& Skrap, M. (2005). Better without (lateral) frontal cortex? Insight problems solved by frontal patients. Brain, 128, 2882-2890.

Russ. S. W. (2001). Primary process thinking and creativity: affect and cognition. Creativity research journal, 13, $27-35$.

Storm, B. C. \& Angello, G. (2010). Overcoming fixation: Creative problem solving and retrieval-induced forgetting. Psychological Science, 21, 1263-1265.

Storm, B. C., \& Patel, T. N. (2014). Forgetting as a consequence and enabler of creative thinking. Journal of Experimental Psychology: Learning, Memory, and Cognition, 40, 15941609.

Vartanian, O. (2009). Variable attention facilitates creative problem solving. Psychology of Aesthetics, Creativity, and the Arts, 3, 57-59.

White. H. A., \& Shah. P. (2006). Uninhibited imaginations : creativity in adults with Attention-Deficit/Hyperactivity Disorder. Personality and Individual Differences, 40, 1121 1131. 
Figure captions

Figure 1: A) Example of trials in the congruent (i.e., the control dual-task condition) and B) the incongruent (i.e., the inhibition dual-task condition) Color Word Stroop task conditions. The text bubbles are examples of verbal responses provided by the participants in the Egg task that could occur at any moment during both the control dual-task and the inhibition dual-task conditions.

Figure 2: A) Scores of fluidity according to the experimental conditions in Experiment 1. B) Scores of fluidity according to the experimental conditions in Experiment 2.

Figure 3: A) Example of trials in the low-demanding control dual-task condition and B) the highdemanding dual-task condition. In addition, the text bubbles are examples of verbal responses provided by the participants in the Egg task that could occur at any moment during during both the low-demanding control dual-task condition and the high-demanding dual-task condition. 
(C) 2017, American Psychological Association. This paper is not the copy of record and may not exactly replicate the final, authoritative version of the article. Please do not copy or cite without authors permission. The final article will be available, upon publication, via its DOI: 10.1037/aca0000140 